Research Article

\title{
Wave Propagation in L-Shape Beams with Piezoelectric Shunting Arrays
}

\author{
Shengbing Chen (D), Yubao Song, and Hao Zhang \\ China Aerodynamics Research and Development Center, Mianyang 621000, China \\ Correspondence should be addressed to Shengbing Chen; nudt_chen@163.com
}

Received 14 November 2018; Revised 28 January 2019; Accepted 26 February 2019; Published 11 April 2019

Academic Editor: Luca Landi

Copyright (c) 2019 Shengbing Chen et al. This is an open access article distributed under the Creative Commons Attribution License, which permits unrestricted use, distribution, and reproduction in any medium, provided the original work is properly cited.

\begin{abstract}
Piezoelectric shunting arrays are employed to control the elastic wave propagation in L-shape beams. Unlike straight beams where longitudinal and flexural waves usually propagate independently, these waves are coupled in an L-shape beam. Based on transfer matrix method and Bloch theorem, dispersion curves and vibration transmissibility are evaluated and analyzed. A locally resonant gap is produced on the flexural and longitudinal waves, respectively, whose locations are nonoverlapped if the shunt damping is void. However, the longitudinal wave band gap can be completely overlaid by the flexural one when a proper shunting resistance is involved. With the decreasing of shunting inductance, the locations of longitudinal and flexural wave gaps both go up to higher frequencies which agree with the variation of resonant frequencies, but they are less affected by shunting resistance. As the resistance increases, the width of the band gaps grows, whereas the attainable maximum attenuation within the band gaps shows a significant decrease. Also, finite element simulations are performed to validate the numerical predictions, which demonstrate that the resulting transmissibility of displacements agree well with the band gaps.
\end{abstract}

\section{Introduction}

Flexible structures are extensively applied in large aerospace products, which represents a trend as well as great challenge work for industrial and academic communities. Due to low structural damping and lack of air drag, these vibrations or elastic waves propagating in the structures are lightly decayed that leads to fatigue, instability, or other problems $[1,2]$. Beam element as one of the principal components of space structures, such as truss and framework, has received considerable attention of engineers and scientists in the noise and vibration control field [3-5]. Therefore, much effort has been exerted on vibration mitigation of flexible beams. Nevertheless, this vibration or elastic wave propagation in the beam-type structures also attracts significant attention, especially when the vibrationless environment is desired, such as payload of high resolution satellites and platform for high accuracy experiments.

In the past two decades, elastic wave propagation in periodic composite materials or structures, named phononic crystals (PCs), has received great attention [6-9]. The primary property of PCs is band-gap behavior, i.e., the propagation of elastic waves whose frequencies lie in the band gap will be forbidden. The seminal work of Liu et al. sparked passionate research interest in locally resonant (LR) PCs [8]. They examined three-dimensional PCs consisting of cubic arrays of coated lead spheres immersed in an epoxy matrix and proposed a new type of band-gap formation mechanism, i.e., locally resonant band gap. More recently, the development of LR PCs has been extended to newly emerging field: acoustic metamaterials [10-12]. Acoustic metamaterials are generally regarded as materials with artificial microstructures that possess unusual physical properties such as band gaps, negative refraction, acoustic cloaking, etc.

Structures periodically shunted by electrical resonant circuits can also produce locally resonant band gaps. As a result, piezoelectric shunting arrays can be utilized to suppress the vibration or elastic wave propagation in flexible beams. Particularly, the band gaps can be tuned to the desired frequencies conveniently via adjustment of the circuit parameters. The original periodic shunting concept 
was proposed by Thorp et al. who utilized an array of shunted piezoelectric patches that mounted on a rod to create band gaps centered at the resonant frequency of the shunting circuits [13]. Wang et al. experimentally investigated the low-frequency locally resonant gaps induced by resonant shunting arrays [14]. Airoldi et al. proposed the idea of producing multiple band gaps in a beam with multiresonant shunts [15]. Also, they designed tunable acoustic metamaterials with periodic arrays of resonant shunts [16]. We also performed some theoretical and experimental investigations on flexible structures with arrays of shunting circuits, and some useful conclusions are arrived $[17,18]$. These studies demonstrate that the equivalent mechanical properties of host structures can be modified by piezoelectric shunts and suggest the application for developing tunable band gaps. On the whole, longitudinal and flexural waves in beams with shunting arrays are generally examined independently. Thus, longitudinal and flexural band gaps are usually analyzed separately. In practical structures, however, different beams commonly join together by welding or thread connection with certain intersection angle to form a framework or truss. Actually, the flexural and longitudinal waves are usually coupled and propagate simultaneously in such beam structures. Hence, the goal of this work is to investigate the elastic wave propagation in an L-shape beam with piezoelectric shunting arrays and to achieve a broadband suppression of flexural and longitudinal vibrations.

In this paper, piezoelectric shunting arrays are periodically bonded to the surfaces of an aluminum L-shape beam to produce a locally resonant band gap over the desired frequency range. Based on the transfer matrix (TM) method and Bloch theorem, numerical investigations are performed in detail. Specifically, the structure configuration and bandgap calculation based on the TM method are described in Section 2. Firstly, layout of the assembled beam is illustrated and effective stress equation of shunted piezoelectric patches is derived. Secondly, transfer matrix is obtained from interface continuity conditions. Then, propagation constant is calculated by applying Bloch periodic conditions to the transfer matrix. Also, vibration transmissibility is derived to quantify the capacity of vibration suppression. Numerical results and discussions are demonstrated in Section 3. The dispersion curves are illustrated, where locally resonant band gap is characterized. Parametric analyses of shunting circuits are carried out, and some rules are summarized. Transmissibility of displacements obtained from the TM method and finite element method (FEM) is compared to verify the theoretical calculations. The variation of vibration transmissibility with intersection angle and shunting parameters is also demonstrated. Finally, conclusions are summarized in Section 4.

\section{Structure Configuration and Mathematical Model}

2.1. Configuration. The assembled composite beam consists of a substrate L-shape beam and two arrays of shunted piezoelectric patches (Figure 1), which are

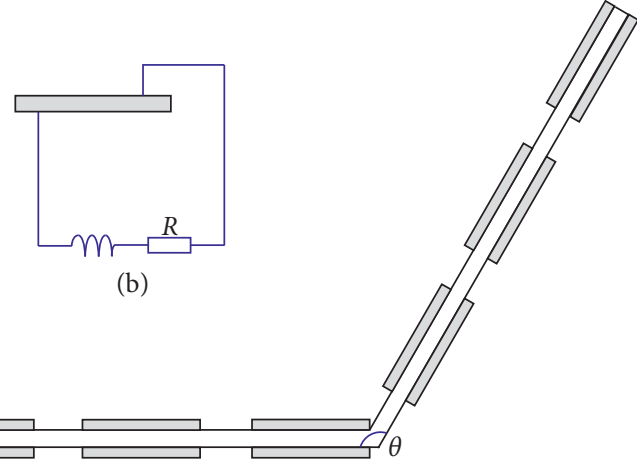

(a)

FIGURE 1: Sketch of the composite beam: (a) L-shape beam with piezoelectric patches and (b) connection pattern of the shunting circuit.

oppositely mounted on the upper and lower surfaces of the beam. Each piezoelectric patch is connected to a resistiveinductive circuit. As a result, the composite beam behaves as a one-dimensional waveguide propagating different waves. For simplicity, we only consider the longitudinal wave and flexural wave in this paper. Apparently, the L-shape beam can be considered as two straight beams joining together with an intersection angle of $\theta$. In addition, the parameters of piezoelectric patches and shunting circuits are identical. Hence, the waveguide will be regarded as superlattice structures, whose wave propagation properties can be conveniently characterized through dynamics analyses of a unit cell (Figure 2) combined with the application of Bloch theorem. The segments without and with piezoelectric patches are denoted as A and B, respectively.

2.2. Modeling of Shunted Piezoelectric Patches. Piezoelectric patches are polarized along with the $z$-axis, whose surfaces are assumed to be free of constraint except those perpendicular to the $x$-axis. For convenience, the directions along with $x, y$, and $z$ axes are denoted by 1,2 , and 3 in the piezoelectric equations, respectively. As a result, the reduced piezoelectric equations are [13]

$$
\left\{\begin{array}{l}
S_{1}=s_{11}^{E} T_{1}+d_{31} E_{3}, \\
D_{3}=d_{31} T_{1}+\varepsilon_{33}^{T} E_{3},
\end{array}\right.
$$

where $S_{1}$ and $T_{1}$ are mechanical strain and stress, respectively; $s_{11}^{E}$ is compliance coefficient at constant electric field; $d_{31}$ is piezoelectric strain constant coupling electrical and mechanical properties; $D_{3}$ is electric displacement on the electrodes; $\varepsilon_{33}^{T}$ is the permittivity at constant stress; and $E_{3}$ is the electric field intensity.

In the equation set (1), eliminating $T_{1}$ gives

$$
D_{3}=\frac{d_{31}}{s_{11}^{E}} S_{1}+\varepsilon_{33}^{S} E_{3},
$$

where $\varepsilon_{33}^{S}$ is the permittivity at constant strain, which can be expressed as 


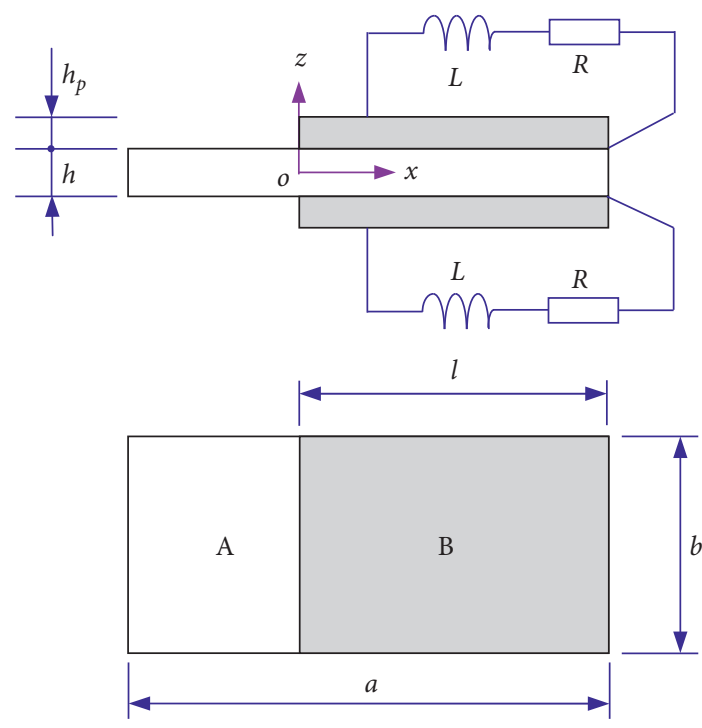

FIgURE 2: Sketch of the unit cell.

$$
\varepsilon_{33}^{S}=\varepsilon_{33}^{T}-\frac{d_{31}^{2}}{s_{11}^{E}} .
$$

The electric charge on the electrodes of each piezoelectric patch can be given by

$$
Q_{s}=\iint_{A_{s}} D_{3} d A
$$

where $A_{s}$ is the area of the electrode and $A_{s}=b l$.

Substituting equation (2) into equation (4) yields

$$
E_{3}=\frac{Q_{s}}{\varepsilon_{33}^{S} A_{s}}-\frac{d_{31} b}{s_{11}^{E} \varepsilon_{33}^{S} A_{s}} \int_{0}^{l} S_{1} d x .
$$

On the other hand, the piezoelectric patch is attached to a resistive-inductive circuit, so applying Ohm's law yields

$$
Q_{s}=-\frac{E_{3} h_{p}}{s(R+L s)},
$$

where $s$ is the complex variable of Laplace transformation.

The beam propagates longitudinal and flexural waves, so mechanical strain in the piezoelectric patch can be written as

$$
S_{1}=\frac{\partial u(x, t)}{\partial x}-\frac{h+h_{p}}{2} \cdot \frac{\partial^{2} w(x, t)}{\partial x^{2}},
$$

where $u(x, t)$ and $w(x, t)$ are longitudinal and flexural displacements, respectively.

Substituting equations (6) and (7) into equation (5) yields

$$
\begin{aligned}
E_{3}= & -\frac{s d_{31} b(R+L s)}{s_{11}^{E} h_{p}\left[s(R+L s) C_{p}^{S}+1\right]}\{u(l, t)-u(0, t) \\
& \left.-\frac{h+h_{p}}{2}\left[\frac{\partial w(l, t)}{\partial x}-\frac{\partial w(0, t)}{\partial x}\right]\right\},
\end{aligned}
$$

where $C_{p}^{S}$ is the capacitance of the piezoelectric patch at constant strain and

$$
C_{p}^{S}=\frac{\varepsilon_{33}^{S} A_{s}}{h_{p}} .
$$

Substituting equation (8) into equation (1) yields

$$
T_{1}=\frac{S_{1}}{s_{11}^{E}}+T_{a}
$$

where

$$
\begin{aligned}
T_{a}= & \frac{s d_{31}^{2} b(R+L s)}{\left(s_{11}^{E}\right)^{2} h_{p}\left[s(R+L s) C_{p}^{S}+1\right]}\{u(l, t)-u(0, t) \\
& \left.-\frac{h+h_{p}}{2}\left[\frac{\partial w(l, t)}{\partial x}-\frac{\partial w(0, t)}{\partial x}\right]\right\} .
\end{aligned}
$$

2.3. Governing Equations of Wave Motion. On the assumption of Euler-Bernoulli beam, the governing equation of longitudinal and flexural motions of the beam element can be given by

$$
\left\{\begin{array}{l}
E_{i} \frac{\partial^{2} u(x, t)}{\partial x^{2}}-\rho_{i} \frac{\partial^{2} u(x, t)}{\partial t^{2}}=0 \\
E_{i} I_{i} \frac{\partial^{4} w(x, t)}{\partial x^{4}}+\rho_{i} A_{i} \frac{\partial^{2} w(x, t)}{\partial t^{2}}=0
\end{array}\right.
$$

where $E_{i}$ is Young's modulus, $\rho_{i}$ is the density, $I_{i}$ is the inertia moment of cross section, and $A_{i}$ is the area of cross section. Here, $i=1,2$, which represent segments $\mathrm{A}$ and $\mathrm{B}$, respectively.

If harmonic motions of the beam are assumed, the longitudinal and transverse displacements can be written as

$$
\left\{\begin{array}{l}
u(x, t)=U(x) \mathrm{e}^{i \omega t} \\
w(x, t)=W(x) \mathrm{e}^{i \omega t}
\end{array}\right.
$$

where $\omega$ is the angular frequency and $i=\sqrt{-1}$.

Substituting equation (13) into equation (12) and solving the differential equations yield

$$
\left\{\begin{array}{c}
U(x)=c_{1} \cos \left(\lambda_{i} x\right)+c_{2} \sin \left(\lambda_{i} x\right), \\
W(x)=c_{3} \cos \left(\gamma_{i} x\right)+c_{4} \sin \left(\gamma_{i} x\right) \\
+c_{5} \cosh \left(\gamma_{i} x\right)+c_{6} \sinh \left(\gamma_{i} x\right),
\end{array}\right.
$$

where $c_{i},(i=1,2, \ldots, 6)$ are undetermined coefficients. $\lambda_{i}$ and $\gamma_{i}$ are wavenumbers of longitudinal and flexural waves, which can be given by

$$
\begin{aligned}
& \lambda_{i}=\sqrt{\frac{\rho_{i}}{E_{i}} \omega}, \\
& \gamma_{i}=\sqrt[4]{\frac{\rho_{i} A_{i} \omega^{2}}{E_{i} I_{i}}} .
\end{aligned}
$$


Therefore, the vector of generalized displacements and forces can be rewritten as

$$
\mathbf{Z}(x)=\mathbf{H}(x) \mathbf{c},
$$

where $\mathbf{Z}(x)=\left[\begin{array}{llllll}U(x) & N(x) & W(x) & \vartheta(x) & M(x) & Q(x)\end{array}\right]^{T}$. $\vartheta(x)$ is the slope of the flexural displacement. $N(x), M(x)$ and $Q(x)$ are tension, moment, and shear force, respectively. $\mathbf{c}=\left[\begin{array}{llllll}c_{1} & c_{2} & c_{3} & c_{4} & c_{5} & c_{6}\end{array}\right]^{T}$ and $\mathbf{H}(x)$ is the coefficient matrix (refer to Appendix for details).

The continuity conditions between segments A and B in the $n$th cell can be expressed as

$$
\mathbf{Z}_{n, \mathrm{~A}}(0)=\mathbf{Z}_{n, \mathrm{~B}}(0) \text {, }
$$

where the first subscript denotes cell number and the second is segment notation.

Similarly, the continuity conditions between $n$th and $(n+1)$ th cells also can be written as

$$
\mathbf{Z}_{n+1, \mathrm{~A}}(l-a)=\mathbf{Z}_{n, \mathrm{~B}}(l) .
$$

Hence, combining equations (17) and (18) gives

$$
\mathbf{c}_{n+1}=\mathbf{T c}_{n},
$$

where $\mathbf{T}$ is the transfer matrix of the unit cell and

$$
\mathbf{T}=\mathbf{H}_{\mathrm{A}}^{-1}(l-a) \mathbf{H}_{\mathrm{B}}(l) \mathbf{H}_{\mathrm{B}}^{-1}(0) \mathbf{H}_{\mathrm{A}}(0) .
$$

On the other hand, applying the Bloch theorem to the periodic beam gives

$$
\mathbf{c}_{n+1}=\mathrm{e}^{\mu} \mathbf{c}_{n},
$$

where $\mu$ is propagation constant, whose real part $\alpha$ and imaginary part $\beta$ are called attenuation constant and phase constant, respectively. If $\alpha=0$, the corresponding wave can propagate without decay; otherwise, the corresponding wave will be attenuated.

Combining equations (19) and (21) yields

$$
\left(\mathbf{T}-\mathrm{e}^{\mu}\right) \mathbf{c}_{n}=0 .
$$

Therefore, the propagation constant can be obtained by solving eigenvalue of the transfer matrix.

2.4. Derivation of Vibration Transmissibility. When the L-shape beam is composed of finite periods, it can be divided into two components (Figure 3), which consists of $M$ and $N$ cells, respectively. The constraint of the beam is free-free and an excitation force $F$ is applied on the left end with incident angle $\delta$. As a result, the boundary conditions of the L-shape beam are summarized as follows:

(1) On the left end

$$
\left\{\begin{array}{l}
N_{1 \mathrm{~L}}=F \cos \delta, \\
M_{1 \mathrm{~L}}=0, \\
Q_{1 \mathrm{~L}}=F \sin \delta .
\end{array}\right.
$$

(2) On the right end

$$
\left\{\begin{array}{l}
N_{2 \mathrm{R}}=0 \\
M_{2 \mathrm{R}}=0 \\
Q_{2 \mathrm{R}}=0
\end{array}\right.
$$

Here, the first subscript is component number, and the second represents location, i.e., on the left end (L) or right end $(\mathrm{R})$.

Substituting equation (14) into equations (23) and (24) and assembling them into matrix form yield

$$
\begin{aligned}
& \boldsymbol{\Psi c}_{1,1}=\left[\begin{array}{c}
F \cos \theta \\
0 \\
F \sin \theta
\end{array}\right], \\
& \boldsymbol{\Phi c}_{2, N}=\left[\begin{array}{l}
0 \\
0 \\
0
\end{array}\right],
\end{aligned}
$$

where the first subscript is component number and the second is cell number.

On the other hand, the right end motion of component 1 can be expressed as

$$
\mathbf{Z}_{1 \mathrm{R}}=\mathbf{H}_{\mathrm{B}}(l) \mathbf{c}_{1, M} \cdot
$$

Substituting equation (19) into equation (27) gives

$$
\mathbf{Z}_{1 \mathrm{R}}=\mathbf{H}_{\mathrm{B}}(l) \cdot \mathbf{T}^{M-1} \mathbf{c}_{1,1} \text {. }
$$

Similarly

$$
\mathbf{Z}_{2 \mathrm{R}}=\mathbf{H}_{\mathrm{B}}(l) \mathbf{c}_{2, N}=\mathbf{H}_{\mathrm{B}}(l) \cdot \mathbf{T}^{N-1} \mathbf{c}_{2,1} \cdot
$$

At the joint of components 1 and 2, it has the relation

$$
\mathbf{Z}_{1 \mathrm{R}}=\Theta \mathbf{Z}_{2 \mathrm{~L}}=\boldsymbol{\Theta} \mathbf{H}_{\mathrm{A}}(l-a) \mathbf{c}_{2,1},
$$

where

$$
\boldsymbol{\Theta}=\left[\begin{array}{cccccc}
-\cos \theta & 0 & -\sin \theta & 0 & 0 & 0 \\
0 & -\cos \theta & 0 & 0 & 0 & -\sin \theta \\
\sin \theta & 0 & -\cos \theta & 0 & 0 & 0 \\
0 & 0 & 0 & 1 & 0 & 0 \\
0 & 0 & 0 & 0 & 1 & 0 \\
0 & \sin \theta & 0 & 0 & 0 & -\cos \theta
\end{array}\right] .
$$

Hence, combining equations (28), (29), and (30) yields

$$
\mathbf{c}_{2, N}=\Pi \mathbf{c}_{1,1} \text {, }
$$

where

$$
\boldsymbol{\Pi}=\mathbf{T}^{N-1}\left[\boldsymbol{\Theta} \mathbf{H}_{\mathrm{A}}(l-a)\right]^{-1} \mathbf{H}_{\mathrm{B}}(l) \cdot \mathbf{T}^{M-1} .
$$

Finally, equations (25), (26), and (32) can be cast into assembled form

$$
\mathbf{M X}=\mathbf{Y}
$$




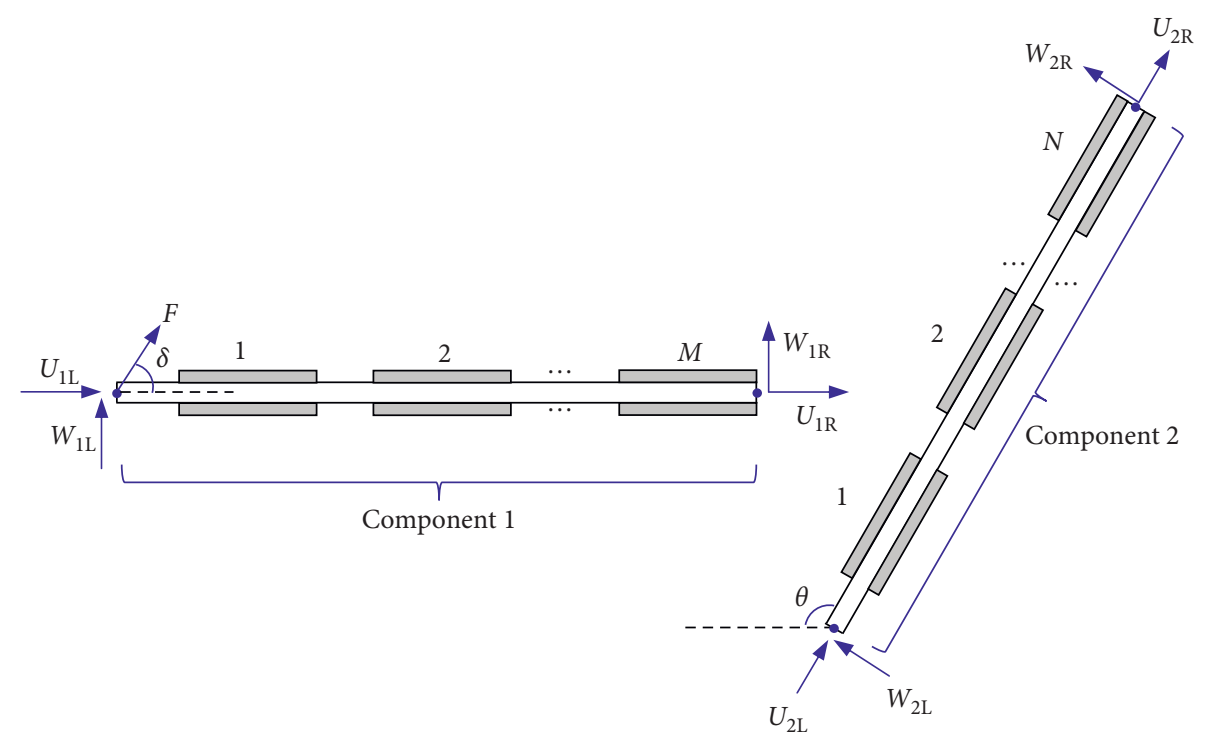

Figure 3: Components of the L-shape beam.

where

$$
\begin{aligned}
\mathbf{M} & =\left[\begin{array}{cc}
\mathbf{\Psi} & \mathbf{0} \\
\mathbf{0} & \boldsymbol{\Phi} \\
\Pi & -\mathbf{I}
\end{array}\right], \\
\mathbf{X} & =\left[\begin{array}{lll}
\mathbf{c}_{1,1} & \mathbf{c}_{2, N}
\end{array}\right], \\
\mathbf{Y} & =\left[\begin{array}{llllllllllll}
d \cos \theta & d \sin \theta & 0 & 0 & 0 & 0 & 0 & 0 & 0 & 0 & 0 & 0
\end{array}\right]^{T},
\end{aligned}
$$

and $\mathbf{I}$ is unit matrix.

The solution of equation (34) is

$$
\mathbf{X}=\mathbf{M}^{-1} \mathbf{Y} \text {. }
$$

Consequently, the longitudinal and flexural displacements at the left and right ends can be solved as

$$
\left[\begin{array}{c}
U_{1 \mathrm{~L}} \\
W_{1 \mathrm{~L}} \\
U_{2 \mathrm{R}} \\
W_{2 \mathrm{R}}
\end{array}\right]=\mathbf{X}^{T} \boldsymbol{\Lambda},
$$

where

$$
\Lambda=\left[\begin{array}{cccc}
\cos \left[\lambda_{1}(l-a)\right] & 0 & \cos \left(\lambda_{2} l\right) & 0 \\
\cos \left[\lambda_{1}(l-a)\right] & 0 & \sin \left(\lambda_{2} l\right) & 0 \\
0 & \cos \left[\gamma_{1}(l-a)\right] & 0 & \cos \left(\gamma_{2} l\right) \\
0 & \sin \left[\gamma_{1}(l-a)\right] & 0 & \sin \left(\gamma_{2} l\right) \\
0 & \cosh \left[\gamma_{1}(l-a)\right] & 0 & \cosh \left(\gamma_{2} l\right) \\
0 & \sinh \left[\gamma_{1}(l-a)\right] & 0 & \sinh \left(\gamma_{2} l\right)
\end{array}\right] .
$$

As the L-shape beam is finite, there will be no energy transmission, if damping is void. Therefore, the vibration transmissibility is defined as the transmissibility of displacements, namely,

$$
\begin{aligned}
& T_{\mathrm{LL}}=20 \lg \left|\frac{U_{2 \mathrm{R}}}{U_{1 \mathrm{~L}}}\right|, \\
& T_{\mathrm{FF}}=20 \lg \left|\frac{W_{2 \mathrm{R}}}{W_{1 \mathrm{~L}}}\right|, \\
& T_{\mathrm{FL}}=20 \lg \left|\frac{U_{2 \mathrm{R}}}{W_{1 \mathrm{~L}}}\right|, \\
& T_{\mathrm{LF}}=20 \lg \left|\frac{W_{2 \mathrm{R}}}{U_{1 \mathrm{~L}}}\right| .
\end{aligned}
$$

Equations (39) and (40) present the displacement transmissibility of longitudinal and flexural waves, while equations (41) and (42) demonstrate the conversions between different wave modes. When the transmissibility is greater than zero, the displacement at the detector end will be larger than that of the source end. Hence, the vibration will be amplified. On the contrary, when the transmissibility is smaller than zero, the displacement at the detector end will be smaller than that of the source end. Hence, the vibration will be decayed.

\section{Numerical Results and Discussions}

As an example, aluminum and PZT-5H are selected as the materials of substrate beam and piezoelectric patches, respectively. The density, Young's modulus, and Poisson's ratio of aluminum are $2700 \mathrm{~kg} / \mathrm{m}^{3}, 70 \mathrm{GPa}$, and 0.3 , respectively. The material parameters of PZT-5H are listed in Table 1. The geometrical parameters are summarized as follows: $l=30 \mathrm{~mm}, b=20 \mathrm{~mm}, a=40 \mathrm{~mm}, h=2 \mathrm{~mm}$, and $h_{p}=0.6 \mathrm{~mm}$. 
TABLE 1: Material parameters of PZT-5H.

\begin{tabular}{lc}
\hline Parameters & Value \\
\hline Density & $7500 \mathrm{~kg} / \mathrm{m}^{-3}$ \\
$s_{11}^{E}$ & $16.5 \times 10^{-12} \mathrm{~m}^{2} / \mathrm{N}$ \\
$d_{31}$ & $-2.74 \times 10^{-10} \mathrm{C} / \mathrm{N}$ \\
$\varepsilon_{33}^{T}$ & $3.01 \times 10^{-8} \mathrm{~F} / \mathrm{m}$ \\
\hline
\end{tabular}

3.1. Dispersion Relations. The band gaps generated in the composite beam are not only produced by local resonances of the shunts but also affected by the shunt damping when electrical resistance is involved. In order to demonstrate the band gaps induced purely by local resonances of the shunting circuits, the electrical resistance $R$ is set to be zero at first. Hence, the propagation constant (when $L=0.4 \mathrm{H}$ ) of the periodic composite beam is illustrated in Figure 4. One locally resonant gap (shaded area in inset) is induced in longitudinal and flexural waves, respectively. The band gap of longitudinal wave is a pure attenuation zone (PAZ) ranging from $1528 \mathrm{~Hz}$ to $1558 \mathrm{~Hz}$, in which the propagation constant is a pure real number. The maximum attenuation occurs at the lower bounding frequency of the band gap, which is a typical feature observed in conventional locally resonant crystals. Also, the phase constant curve near the lower bounding frequency is rather flat, which means that the group velocity of longitudinal wave is close to zero. However, the band gap of flexural wave shows great difference. It is the combination of a pure attenuation zone (PAZ) and a complex attenuation zone (CAZ), whose propagation constant is a complex number. Unlike longitudinal wave which owns one single wave mode, the flexural wave is more complicated which possesses two wave modes, i.e., propagation wave and evanescent wave. Generally, the evanescent wave is neglected when the waveguide is large enough to decay it quickly. However, there is an exception when a CAZ is produced by the coupling effect of the propagation wave and evanescent wave. In the CAZ, the propagation wave and evanescent wave are coupled together and transfer energy to each other. This is the reason why the propagation constant is complex in the CAZ, though no damping is involved. Actually, there is no vibration energy transmitting between cells in the CAZ [19]. The resulting band gap of flexural wave is located in a lower frequency scope, ranging from $1465 \mathrm{~Hz}$ to $1521 \mathrm{~Hz}$. Obviously, the maximum attenuation does not appear at the lower bounding frequency but coincides with the interface of PAZ and CAZ instead. In addition, the phase constant curve near the lower bounding frequency is not as flat as that of the longitudinal wave. That is to say, in the flexural wave band gap, some of the vibration energy is easier to be confined in the matrix, rather than almost completely restricted to the vibrators. Though both the flexural wave band gap and longitudinal wave band gap are induced by the same resonant circuits, the locations of the two band gaps are not overlapped. The reason is that the dynamic capacitance of a piezoelectric patch is variable with constraint conditions. In other words, the resonance frequencies of shunting circuits are different between longitudinal and flexural cases. The actual resonance frequency is affected by the motion of the

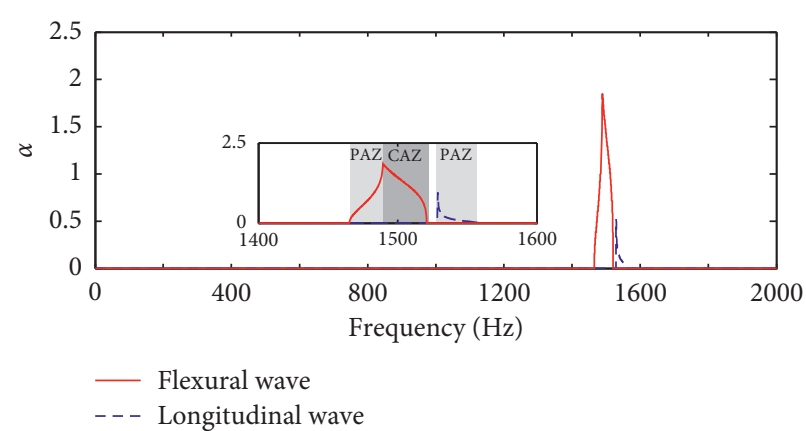

(a)

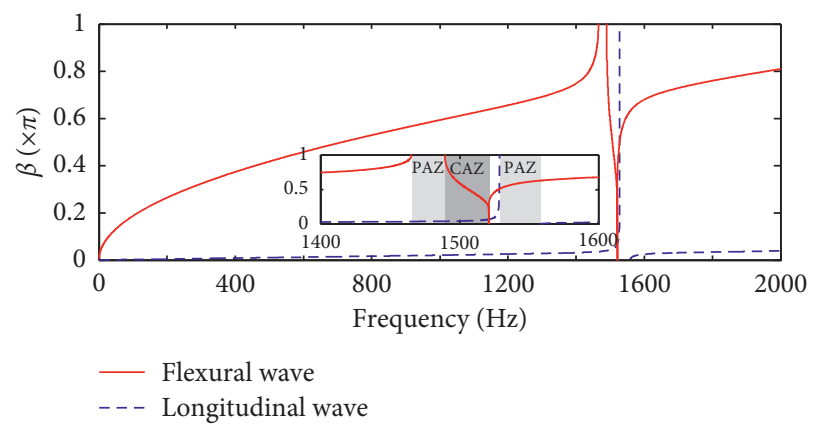

(b)

Figure 4: Propagation constant of the composite beam.

substrate. Generally, the real effective capacitance lies between the capacitance at constant strain and that at constant stress, i.e.,

$$
C_{\text {eff }} \in\left(C_{p}^{S}, C_{p}^{T}\right)
$$

The variations of longitudinal wave gap (LG) and flexural wave gap (FG) with shunting inductance are illustrated in Figure 5. With the decreasing of inductance $L$, the locations of LG and FG both go up to higher frequencies. It is aforementioned that locally resonant gap is produced by local resonance of scatters and thus coincides with the resonance frequency of local vibrators. As for electrical resonators, the resonance frequency is

$$
f_{r}=\frac{1}{2 \pi \sqrt{L C_{\text {eff }}}} .
$$

Evidently, equation (44) also demonstrates that the frequency of band gaps will increase with the decreasing of $L$. Moreover, it is observed that the two band gaps (LG and FG) keep distinct and nonoverlapping.

The effect of shunt damping $(R=100 \Omega)$ on the band gaps is demonstrated in Figure 6. The impacts on longitudinal and flexural wave band gaps are quite similar. Firstly, the attainable maximum attenuation within the band gaps shows a significant decrease when shunting resistance is involved. Evidently, the reason is that local resonance of oscillators will be suppressed by resistance damping. In addition, the phase fluctuations within the band gaps become much smoother. Secondly, the damping effect at the bounding frequencies of band gaps is substantial, resulting in much broader attenuation zones than those 


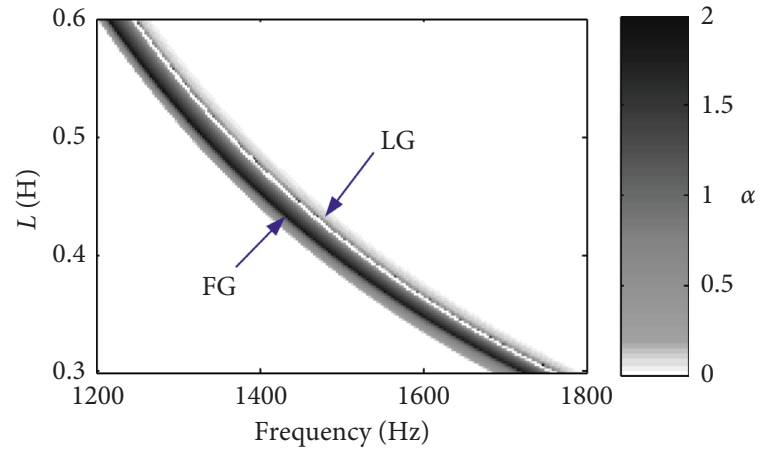

Figure 5: Variation of band gaps with shunting inductance.

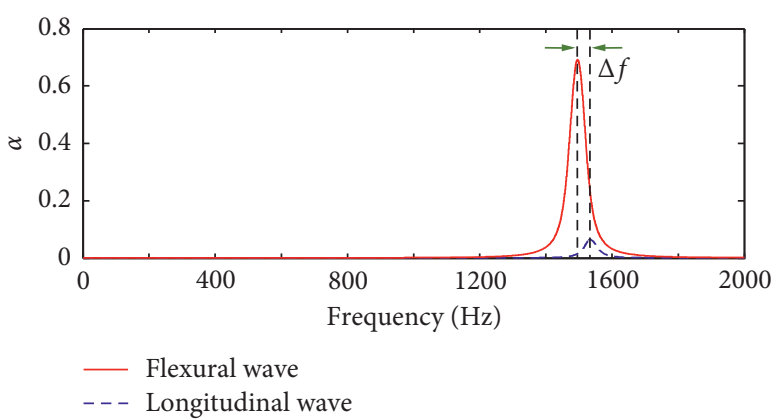

(a)

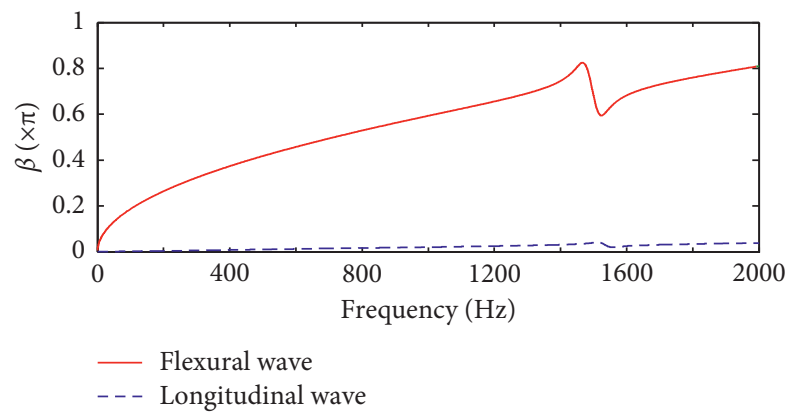

(b)

FIgURE 6: Propagation constant with shunt damping.

merely induced by local resonances. Moreover, the damping capacities at the upper and lower bounding frequencies are similar in magnitude. It is worthy of note that flexural and longitudinal wave band gaps become overlapped. Nevertheless, the frequencies corresponding to the maximum attenuation constant are inconsistent, whose difference $\Delta f$ is about $39 \mathrm{~Hz}$.

The variation of band gaps with shunting resistance $(L=0.4 \mathrm{H})$ is shown in Figure 7. The location of band gaps keeps stationary, while the band width increases with the increase of resistance $R$. This is also in accordance with equation (42), which demonstrates that the resonance frequency is unaffected by shunting resistance. In addition, as the shunting resistance increases, longitudinal and flexural wave gaps are gradually overlapped. For example, the two band gaps will be partially overlapped when $R=20 \Omega$ (see inset of Figure 7).

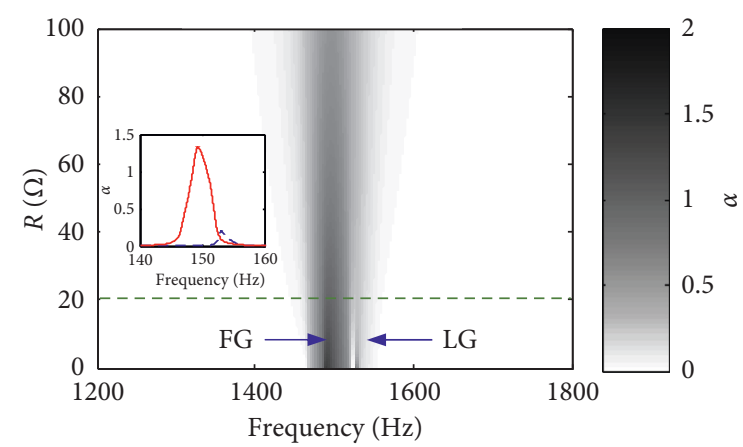

FIGURE 7: Variation of band gaps with shunting resistance.

3.2. Vibration Transmissibility. Finite element method (FEM) is employed to validate the theoretical calculation of TM method. An L-shape beam whose intersection angle $\theta=90^{\circ}$ is selected as the benchmark model. Both two components of the beam consist of three cells, i.e, $M=N=3$, which are built in commercial FEM software COMSOL as shown in Figure 8. The substrate beam and piezoelectric patches are meshed with quadratic hexahedral elements, while the shunting circuits are defined in SPICE circuit editor. An excitation force $F$ of unit amplitude is applied on the left end of the beam, whose incident angle $\delta$ is $45^{\circ}$. The analysis type is frequency response, and direct SPOOLES is selected as the linear system solver.

The comparison between finite element simulation and theoretical calculation is illustrated in Figure 9. The vibration transmissibility without shunting effect, i.e., the circuits are open, is presented in Figures 9(a) and 9(b). On the transmission curves of flexural wave, five peaks appear in the frequency range of $0 \sim 2000 \mathrm{~Hz}$. In contrast, two transmission dips and three peaks occur on the transmission curves of longitudinal wave. A peak denotes that the displacement of the detector end is much larger than that of the source end. So, the beam strongly amplifies the vibration corresponding to the peak. On the contrary, a dip denotes that the displacement of the detector end is much smaller than that of the source end. That is to say, the beam strongly decays the vibration corresponding to the dip. When the shunting circuits are functioning, the two flexural wave transmission peaks (Figure 9(c)) around the band-gap location are suppressed and become much smoother. That is to say, the shunting arrays can effectively decay the flexural wave that falls in the band gap. Similarly, the shunting arrays can also smooth the transmission dips (Figure 9(d)) at the location of the band gap of longitudinal wave. On the whole, the theoretical result by TM method and numerical result by FEM match well, whose small difference may be caused by different modeling postulates. The model of FEM is based on two-dimensional plane-stress assumption, whereas the model of TM method is based on Euler-Bernoulli beam assumption.

The variation of vibration transmissibility with intersection angle is demonstrated in Figure 10, which includes three cases, i.e., acute angle $\left(\theta=60^{\circ}\right)$, blunt angle $\left(\theta=120^{\circ}\right)$, and flat angle $\left(\theta=180^{\circ}\right)$. For comparison, the 


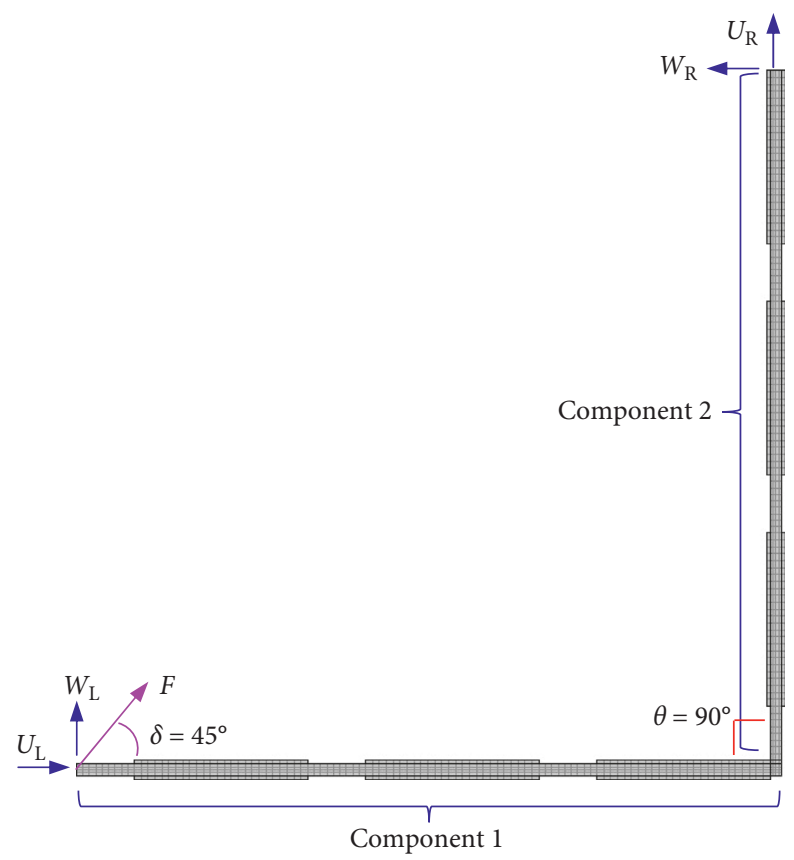

FIGURE 8: Finite element model.

attenuation constants with closed shunting circuits are illustrated in Figures 10(a) and 10(b). As the shunting resistance is rather large $(R=800 \Omega)$, the attenuation zones of flexural and longitudinal waves are very wide with the compromise of a smaller decay magnitude. On the whole, the variation of vibration transmissibility with intersection angle is dramatic. Not only the location of transmission peaks and dips but also the shape of curves changes a lot. However, the invariable is that the attenuation zones on the transmission curves keep stationary, which always coincide with the location of band gaps. Note that the flexural and longitudinal waves are coupled when $\theta=60^{\circ}$ and $\theta=120^{\circ}$. Actually, when the intersection angle is not flat, the longitudinal wave and flexural wave are coupled, and longitudinal waves can be converted to flexural waves whose attenuation effect is stronger. Consequently, the attenuation on vibration transmissibility of longitudinal waves is still substantial, even though the attenuation constant of longitudinal wave is small. When $\theta=180^{\circ}$, the beam becomes straight, so the flexural and longitudinal waves will be decoupled. The attenuation of flexural wave induced by shunting arrays remains distinct as shown in Figure $10(\mathrm{~g})$. In contrast, the influence of shunting circuits on vibration transmissibility of longitudinal wave is less notable (Figure 10(h)). The reason for this is that the attenuation constant of longitudinal wave is much smaller and there is no transmission peak located within the frequency scope of band gap. In a word, the attenuation effect of shunting arrays is always remarkable with different intersection angles, particularly when the angle is not flat.

The variation of vibration transmissibility with circuit parameters is illustrated in Figure 11. The attenuation effect of shunting circuit is weak when the frequency is far away from the band gap, so the graphs are merely plotted over the range of $1000 \sim 2000 \mathrm{~Hz}$. Figures 11(a) and 11(b) demonstrate the variation of transmission curves with shunting resistance. The effect of shunting circuits is apparent. Compared with the open circuits (OC), the two transmission peaks of flexural wave are squeezed away from each other when the circuits are closed. The reason is that resonant shunting circuit will reduce and increase the effective modulus of the piezoelectric patches before and after the resonant frequency, respectively [20]. Note that the shunting circuits generate a new peak on the longitudinal transmission curve. The peak is induced by resonance of shunting circuits, which should coincide with the bounding frequency of band gaps. With the increasing of resistance $R$, the transmission peaks become lower, while the transmission dips get higher. The reason is that the shunt damping will increase with resistance and suppress the resonant oscillation of shunting circuits. However, the influence on locations is small because the resonant frequency of shunting circuit is less affected by resistance. Figures 11(c) and 11(d) demonstrate the variation of transmission curves with shunting inductance. It is observed that the location of transmission trough is significantly affected by the inductance $L$. As the inductance increases, the trough goes to a lower frequency. The reason is that the resonant frequency (refer to equation (44)) of shunting circuits will decrease with the increase of inductance $L$. This rule agrees well with the result in Figure 5.

3.3. Mode Conversion. The longitudinal and flexural waves are coupled (when $\theta<180^{\circ}$ ), so the two wave modes can convert to each other. In order to characterize the mode conversion from longitudinal wave to flexural wave, the incident angle is set to be zero $(\delta=0)$. Hence, there is only 


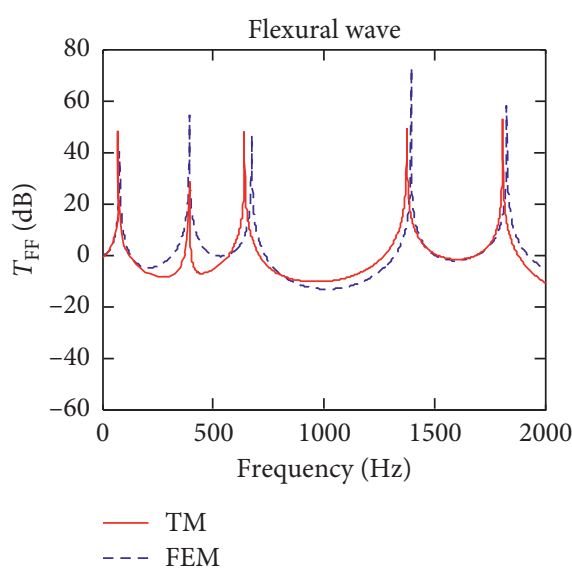

(a)

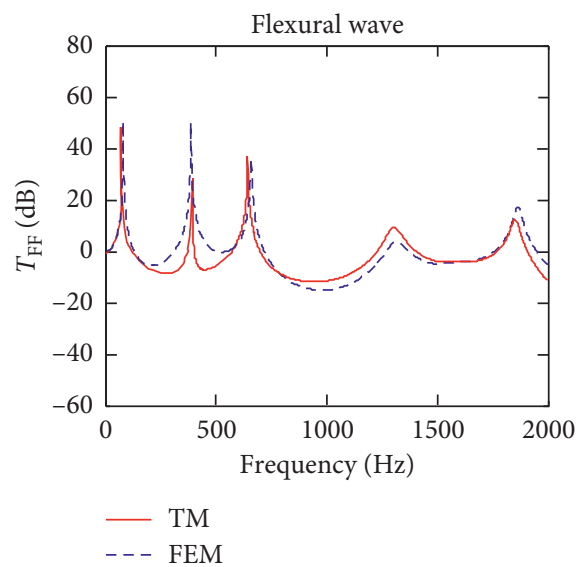

(c)

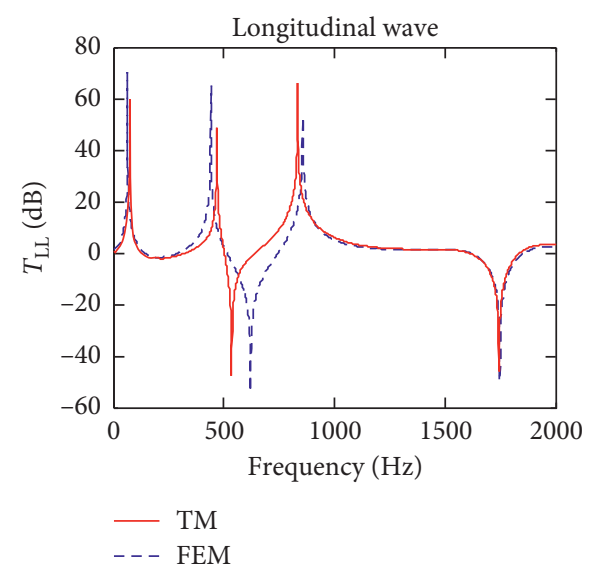

(b)

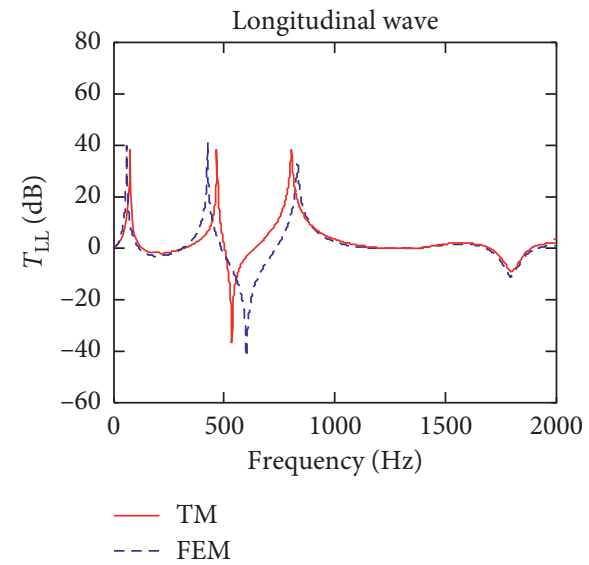

(d)

FIGURE 9: Comparison between TM method and FEM. (a, b) Vibration transmissibility of flexural and longitudinal waves with open circuits. (c, d) Vibration transmissibility of flexural and longitudinal waves with closed circuits, where $L=0.4 \mathrm{H}$ and $R=800 \Omega$.
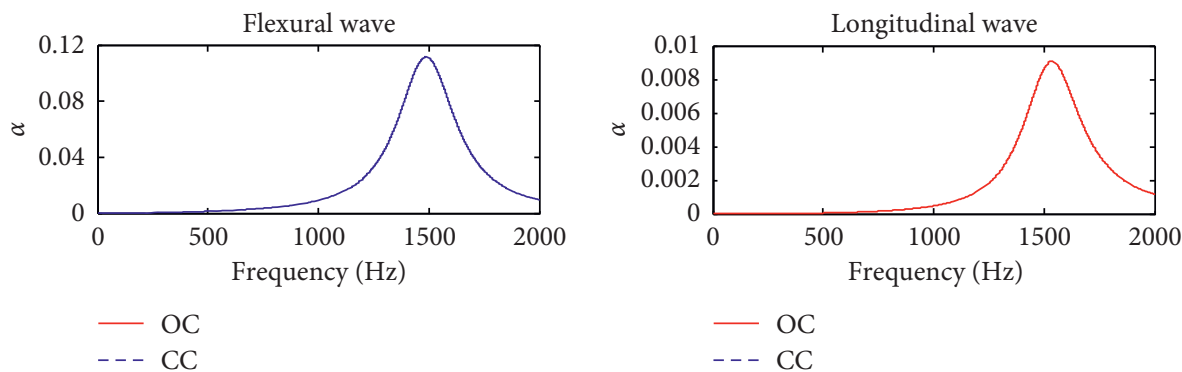

- OC

$---\mathrm{CC}$

(a)

(b)
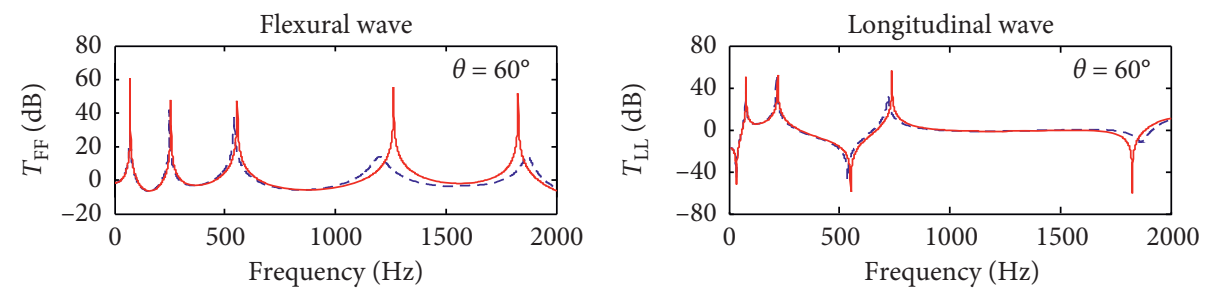

- OC

- OC

$---\mathrm{CC}$

--- CC

(c)

(d)

Figure 10: Continued. 

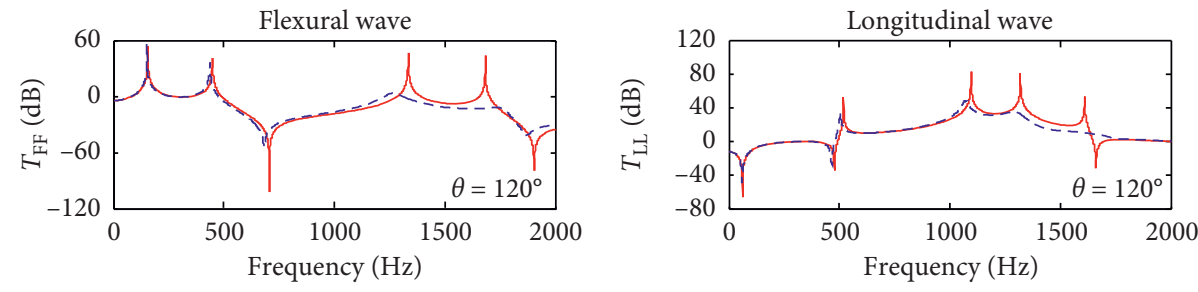

$-\mathrm{OC}$
$---\mathrm{CC}$

- OC

(e)

(f)
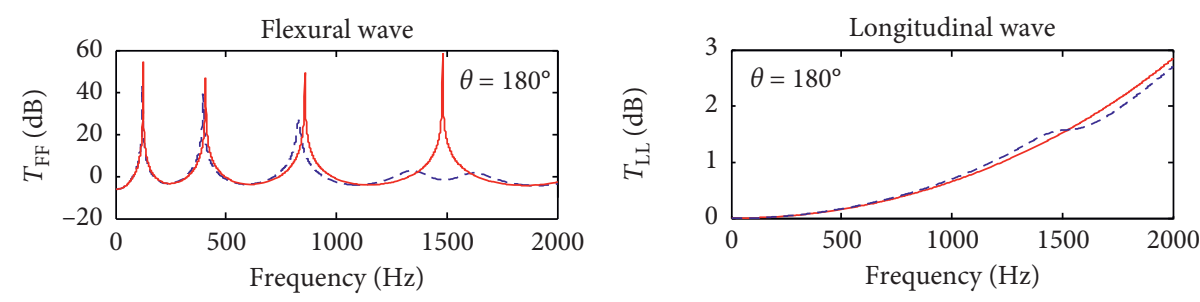

- OC

- $\mathrm{OC}$

- - CC

- - CC

(g)

(h)

FIGURE 10: Variation of vibration transmissibility with intersection angle $\theta$. For comparison, both attenuation constants and vibration transmissibility of flexural and longitudinal waves with open circuits (OC) and closed circuits (CC, $L=0.4 \mathrm{H}$ and $R=800 \Omega$ ) are presented.

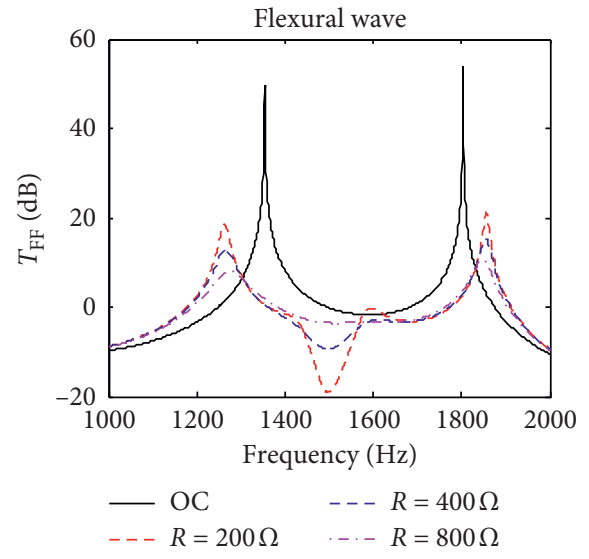

(a)

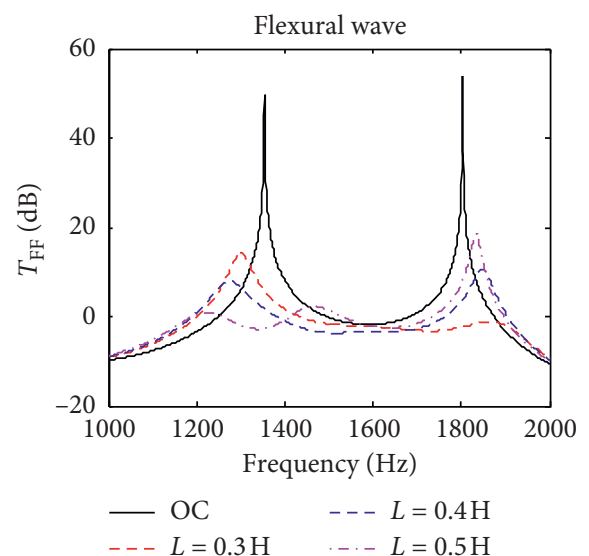

(c)

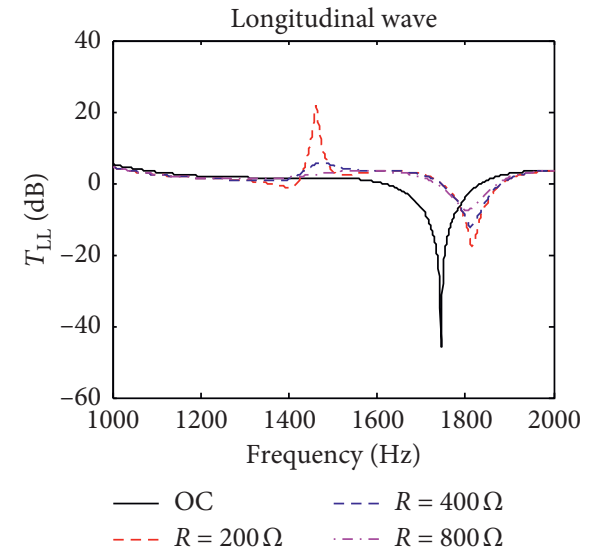

(b)

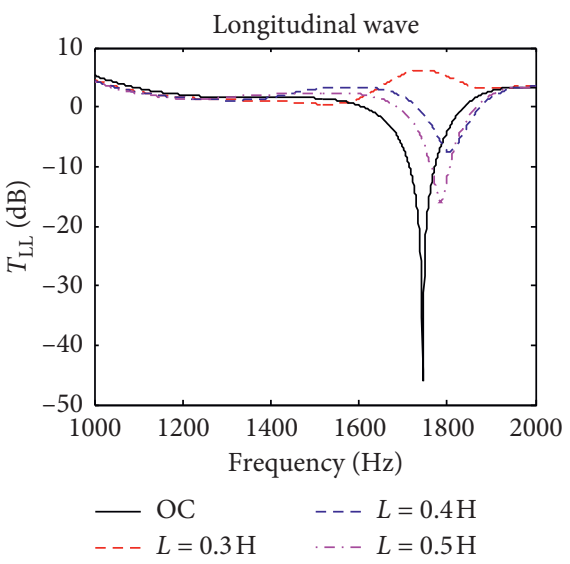

(d)

FIgURE 11: Variation of vibration transmissibility with circuit parameters. $L=0.4 \mathrm{H}$ in $(\mathrm{a}, \mathrm{b}) . R=800 \Omega$ in (c, d). 

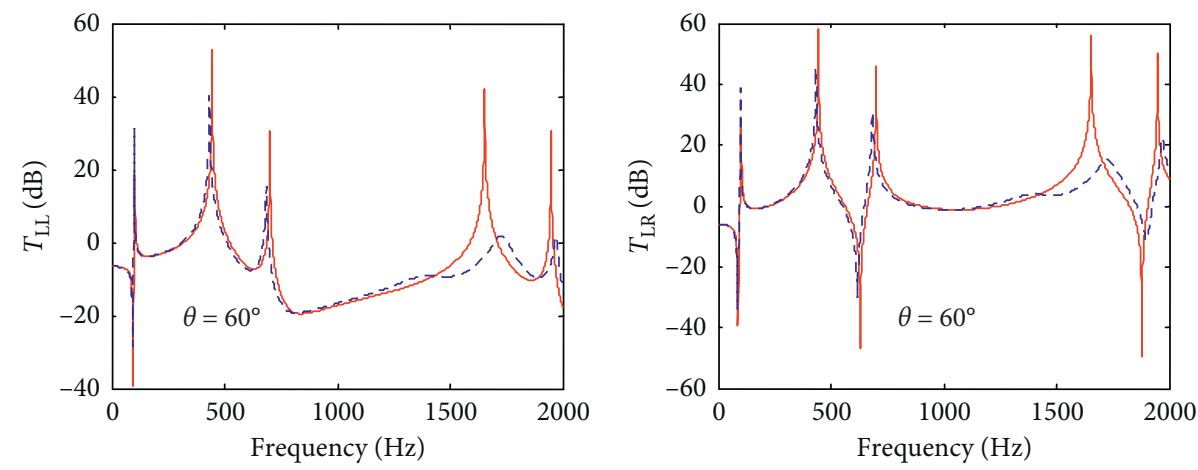

$$
\begin{array}{ll}
- \text { OC } & - \text { OC } \\
-- \text { CC } & -- \text { CC }
\end{array}
$$

(a)

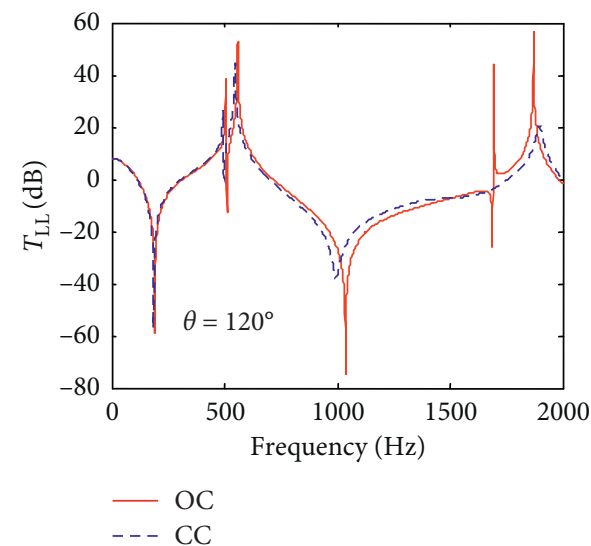

(c)

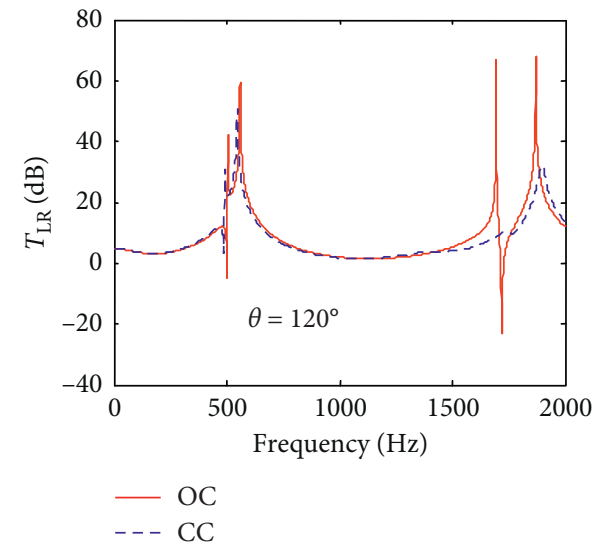

(d)

Figure 12: Mode conversion from longitudinal to flexural waves with open circuits (OC) and closed circuits (CC, $L=0.4 \mathrm{H}$ and $R=800 \Omega$ ).

longitudinal wave excitation. The vibration transmissibility is plotted in Figure 12. Specifically, the transmissibility of longitudinal wave is demonstrated in Figures $12(\mathrm{a})$ and $12(\mathrm{c})$ with different intersection angles $\left(\theta=60^{\circ}\right.$ and $\left.\theta=120^{\circ}\right)$, respectively. The characterization of longitudinal wave converted to flexural wave is presented in Figures 12(b) and 12(d). It is observed that flexural wave has been produced, though the excitation force is fully longitudinal. Both the longitudinal wave and the converted flexural wave are decayed by the shunting circuits (CC). With different intersection angles (acute and blunt), the attenuation of shunt circuits is rather similar.

Similarly, to characterize the conversion from flexural wave to longitudinal wave, the incident angle is set to be right angle $\left(\delta=90^{\circ}\right)$. Therefore, there is merely flexural wave excitation. The vibration transmissibility is presented in Figure 13. The transmissibility of flexural wave is demonstrated in Figures 13(a) and 13(c) with different intersection angles $\left(\theta=60^{\circ}\right.$ and $\left.\theta=120^{\circ}\right)$, respectively. The characterization of flexural wave converted to longitudinal wave is presented in Figures 13(b) and 13(d). One can find that longitudinal wave has also been induced, though the excitation force is completely flexural. Also, both the flexural wave and the converted longitudinal wave are simultaneously decayed by the shunting circuits (CC). However, some of the transmission peaks of longitudinal wave are not fully developed.

In a word, the flexural wave and longitudinal wave propagating in the L-shape beam are coupled and coexisting, even though the excitation at the source end is single mode.

\section{Conclusions}

Band gaps and vibration transmissibility of the proposed composite beam which consists of an aluminum L-shape beam and periodic arrays of shunted piezoelectric patches are analyzed theoretically. It is found that a locally resonant band gap is generated on the flexural and longitudinal waves, respectively. The two band gaps are not overlapped if the shunt damping is void. Though they are induced by the same resonant circuits, the dynamic capacitance of a piezoelectric patch is variable with the boundary constraints. With the decreasing of shunting inductance, the locations of longitudinal and flexural wave gaps both go up to higher frequencies, which agree with the variation of resonant frequencies. When a proper shunting resistance is involved, the two band gaps can be completely overlapped. However, the attainable maximum attenuation within the band gaps shows a significant decrease. Finite element simulations are performed to validate the numerical 

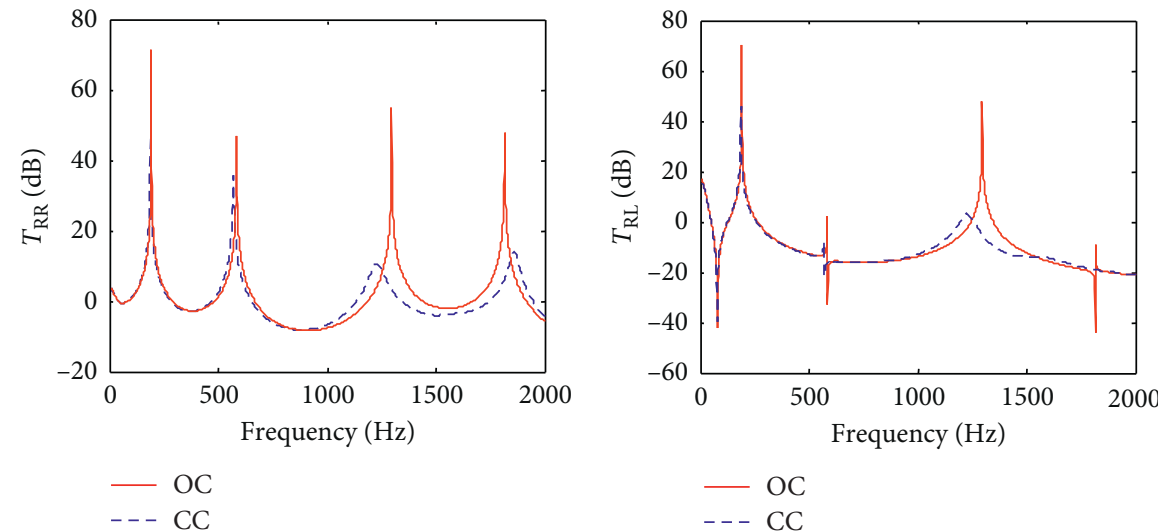

(a)

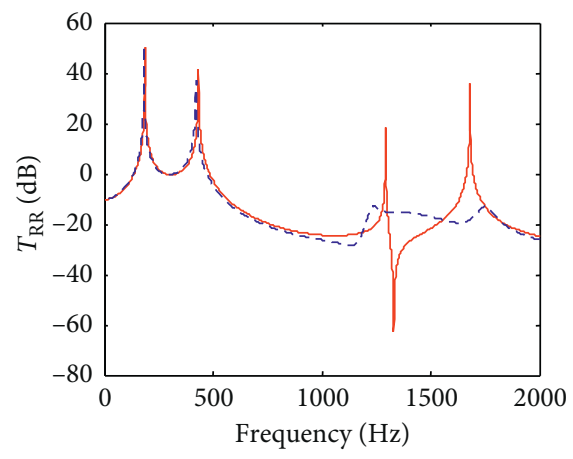

(b)

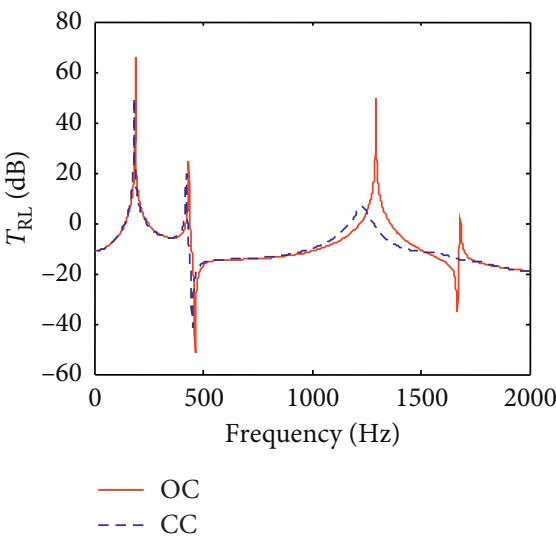

(c)

(d)

FIgURE 13: Mode conversion from flexural to longitudinal waves with open circuits (OC) and closed circuits $(\mathrm{CC}, L=0.4 \mathrm{H}$ and $R=800 \Omega$ ).

predictions, which demonstrate that the resulting vibration transmissibility agrees well with the band gaps. Although the variation of vibration transmissibility with intersection angle is dramatic, the attenuation zones on the transmission curves keep stationary, which always coincide with the location of band gaps. In comparison with the straight beam, the attenuation of longitudinal vibration in the L-shape beam is more efficient because part of the longitudinal vibration will be transformed into flexural vibration, whose attenuation constant is much larger. In summary, piezoelectric shunting arrays can generate a broadband locally resonant gap and effectively decay the flexural and longitudinal waves propagating in the L-shape beam.

\section{Appendix}

The coefficient matrix $\mathbf{H}(x)$ of segment A (without shunted piezopatches) is

$$
\mathbf{H}_{\mathrm{A}}(x)=\left[\begin{array}{cc}
\mathbf{L}_{\mathrm{A}} & \mathbf{0}_{2 \times 4} \\
\mathbf{0}_{4 \times 2} & \mathbf{F}_{\mathrm{A}}
\end{array}\right],
$$

where

$$
\begin{aligned}
\mathbf{L}_{\mathrm{A}} & =\left[\begin{array}{cc}
\cos \left(\lambda_{1} x\right) & \sin \left(\lambda_{1} x\right) \\
-E_{1} A_{1} \lambda_{1} \sin \left(\lambda_{1} x\right) & E_{1} A_{1} \lambda_{1} \cos \left(\lambda_{1} x\right)
\end{array}\right], \\
\mathbf{F}_{\mathrm{A}} & =\left[\begin{array}{cccc}
\cos \left(\gamma_{1} x\right) & \sin \left(\gamma_{1} x\right) & \cosh \left(\gamma_{1} x\right) & \sinh \left(\gamma_{1} x\right) \\
-\gamma_{1} \sin \left(\gamma_{1} x\right) & \gamma_{1} \cos \left(\gamma_{1} x\right) & \gamma_{1} \sinh \left(\gamma_{1} x\right) & \gamma_{1} \cosh \left(\gamma_{1} x\right) \\
-E_{1} I_{1} \gamma_{1}^{2} \cos \left(\gamma_{1} x\right) & -E_{1} I_{1} \gamma_{1}^{2} \sin \left(\gamma_{1} x\right) & E_{1} I_{1} \gamma_{1}^{2} \cosh \left(\gamma_{1} x\right) & E_{1} I_{1} \gamma_{1}^{2} \sinh \left(\gamma_{1} x\right) \\
E_{1} I_{1} \gamma_{1}^{3} \sin \left(\gamma_{1} x\right) & -E_{1} I_{1} \gamma_{1}^{3} \cos \left(\gamma_{1} x\right) & E_{1} I_{1} \gamma_{1}^{3} \sinh \left(\gamma_{1} x\right) & E_{1} I_{1} \gamma_{1}^{3} \cosh \left(\gamma_{1} x\right)
\end{array}\right] .
\end{aligned}
$$


The coefficient matrix $\mathbf{H}(x)$ of segment B (with shunted piezopatches) is

$$
\mathbf{H}_{\mathrm{B}}(x)=\left[\begin{array}{cc}
\mathbf{L}_{\mathrm{B}} & \mathbf{0}_{2 \times 4} \\
\mathbf{0}_{4 \times 2} & \mathbf{F}_{\mathrm{B}}
\end{array}\right]+\left[\begin{array}{c}
\mathbf{0}_{1 \times 6} \\
\mathbf{N}_{\mathrm{S}} \\
\mathbf{0}_{1 \times 6} \\
\mathbf{0}_{1 \times 6} \\
\mathbf{M}_{\mathrm{S}} \\
\mathbf{0}_{1 \times 6}
\end{array}\right],
$$

where

$$
\begin{aligned}
& \mathbf{L}_{\mathrm{B}}=\left[\begin{array}{cc}
\cos \left(\lambda_{2} x\right) & \sin \left(\lambda_{2} x\right) \\
-E_{2} A_{2} \lambda_{2} \sin \left(\lambda_{2} x\right) & E_{2} A_{2} \lambda_{2} \cos \left(\lambda_{2} x\right)
\end{array}\right] \text {, } \\
& \mathbf{F}_{\mathrm{B}}=\left[\begin{array}{cccc}
\cos \left(\gamma_{2} x\right) & \sin \left(\gamma_{2} x\right) & \cosh \left(\gamma_{2} x\right) & \sinh \left(\gamma_{2} x\right) \\
-\gamma_{2} \sin \left(\gamma_{2} x\right) & \gamma_{2} \cos \left(\gamma_{2} x\right) & \gamma_{2} \sinh \left(\gamma_{2} x\right) & \gamma_{2} \cosh \left(\gamma_{2} x\right) \\
-E_{2} I_{2} \gamma_{2}^{2} \cos \left(\gamma_{2} x\right) & -E_{2} I_{2} \gamma_{2}^{2} \sin \left(\gamma_{2} x\right) & E_{2} I_{2} \gamma_{2}^{2} \cosh \left(\gamma_{2} x\right) & E_{2} I_{2} \gamma_{2}^{2} \sinh \left(\gamma_{2} x\right) \\
E_{2} I_{2} \gamma_{2}^{3} \sin \left(\gamma_{2} x\right) & -E_{2} I_{2} \gamma_{2}^{3} \cos \left(\gamma_{2} x\right) & E_{2} I_{2} \gamma_{2}^{3} \sinh \left(\gamma_{2} x\right) & E_{2} I_{2} \gamma_{2}^{3} \cosh \left(\gamma_{2} x\right)
\end{array}\right] \text {, } \\
& \mathbf{N}_{S}=2 k_{s} b h_{p}\left[\cos \left(\lambda_{2} l\right) \sin \left(\lambda_{2} l\right) \quad 0 \quad 0 \quad 0 \quad 0\right]
\end{aligned}
$$

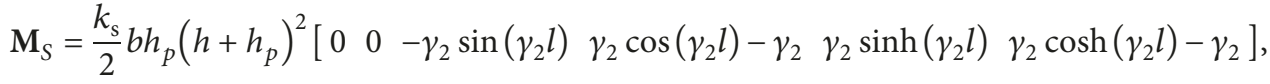

$$
\begin{aligned}
& k_{\mathrm{s}}=\frac{\mathrm{i} \omega d_{31}^{2} b(R+\mathrm{i} \omega L)}{\left(s_{11}^{E}\right)^{2} h_{p}\left[\mathrm{i} \omega(R+\mathrm{i} \omega L) C_{p}^{S}+1\right]} .
\end{aligned}
$$

\section{Data Availability}

The data used to support the findings of this study are available from the corresponding author upon request.

\section{Conflicts of Interest}

The authors declare that they have no conflicts of interest.

\section{Acknowledgments}

This work was funded by the National Natural Science Foundation of China (grant no. 11702306) and also supported by the FengLei Youth Innovation Fund of CARDC (grant no. FLYIF201725).

\section{References}

[1] L. Sirota and Y. Haleve, "Free response and absolute vibration suppression of second-order flexible structures-the traveling wave approach," Journal of Vibration and Acoustics, vol. 132, no. 3, article $031008,2010$.

[2] P. Ambrosio, G. Cazzulani, F. Resta, and F. Ripamonti, "An optimal vibration control logic for minimising fatigue damage in flexible structures," Journal of Sound and Vibration, vol. 333, no. 5, pp. 1269-1280, 2014.

[3] R. Krishna and P. M. Bainum, "Effect of solar radiation disturbance on a flexible beam in orbit," AIAA Journal, vol. 22, no. 5, pp. 677-682, 1984.

[4] C. Seung-Bok and C. Chae-Cheon, "Vibration control of a flexible beam using shape memory alloy actuators," Journal of Guidance, Control, and Dynamics, vol. 19, no. 5, pp. 11781180, 1996.

[5] L.-X. Chen and J.-Q. Sun, "Multi-objective optimal design and experimental validation of tracking control of a rotating flexible beam," Journal of Sound and Vibration, vol. 333, no. 19, pp. 4415-4426, 2014.

[6] M. S. Kushwaha, P. Halevi, G. Martínez, L. Dobrzynski, and B. Djafari-Rouhani, "Theory of acoustic band structure of periodic elastic composites," Physical Review B, vol. 49, no. 4, pp. 2313-2322, 1994.

[7] M. M. Sigalas and E. N. Economou, "Elastic and acoustic wave band structure," Journal of Sound and Vibration, vol. 158, no. 2, pp. 377-382, 1992.

[8] Z. Liu, X. Zhang, Y. Mao, Y. Y. Zhu, C. T. Yang, and P. Sheng, "Locally resonant sonic materials," Science, vol. 289, no. 5485, pp. 1734-1736, 2000.

[9] Y. Xiao, J. Wen, and X. Wen, "Broadband locally resonant beams containing multiple periodic arrays of attached 
resonators," Physics Letters A, vol. 376, no. 16, pp. 1384-1390, 2012.

[10] J. Li and C. T. Chan, "Double-negative acoustic metamaterial," Physical Review E, vol. 70, no. 5, article 055602, 2004.

[11] N. Fang, D. Xi, J. Xu et al., "Ultrasonic metamaterials with negative modulus," Nature Materials, vol. 5, no. 6, pp. 452456, 2006.

[12] G. W. Milton, "New metamaterials with macroscopic behavior outside that of continuum elastodynamics," New Journal of Physics, vol. 9, no. 10, p. 359, 2007.

[13] O. Thorp, M. Ruzzene, and A. Baz, "Attenuation and localization of wave propagation in rods with periodic shunted piezoelectric patches," Smart Materials and Structures, vol. 10, no. 5, pp. 979-989, 2001.

[14] G. Wang, S. Chen, and J. Wen, "Low-frequency locally resonant band gaps induced by arrays of resonant shunts with Antoniou's circuit: experimental investigation on beams," Smart Materials and Structures, vol. 20, no. 1, article 015026, 2011.

[15] L. Airoldi and M. Ruzzene, "Wave propagation control in beams through periodic multi-branch shunts," Journal of Intelligent Material Systems and Structures, vol. 22, no. 14, pp. 1567-1579, 2011.

[16] L. Airoldi and M. Ruzzene, "Design of tunable acoustic metamaterials through periodic arrays of resonant shunted piezos," New Journal of Physics, vol. 13, no. 11, article 113010, 2011.

[17] S. Chen, J. Wen, G. Wang, D. Yu, and X. Wen, "Improved modeling of rods with periodic arrays of shunted piezoelectric patches," Journal of Intelligent Material System and Structures, vol. 23, no. 14, pp. 1613-1621, 2012.

[18] S. Chen, G. Wang, J. Wen, and X. Wen, "Wave propagation and attenuation in plates with periodic arrays of shunted piezo-patches," Journal of Sound and Vibration, vol. 332, no. 6, pp. 1520-1532, 2013.

[19] D. J. Mead, "Wave propagation and natural modes in periodic systems: II. Multi-coupled systems, with and without damping," Journal of Sound and Vibration, vol. 40, no. 1, pp. 19-39, 1975.

[20] S. Chen, "Wave propagation in acoustic metamaterials with resonantly shunted cross-shape piezos," Journal of Intelligent Material Systems and Structures, vol. 29, no. 13, pp. 27442753, 2018. 


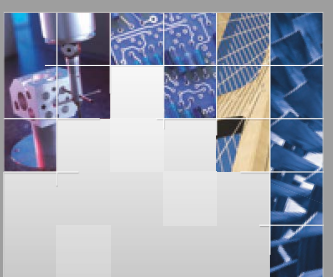

\section{Enfincering}
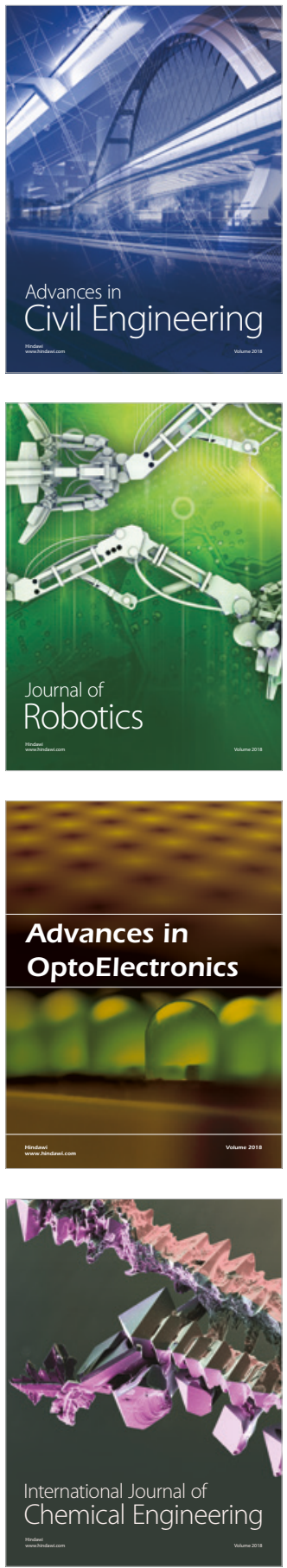

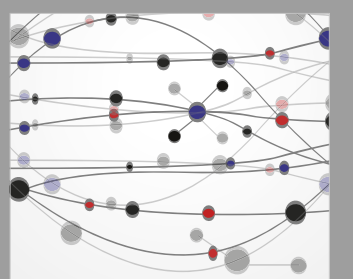

\section{Rotating \\ Machinery}

The Scientific World Journal

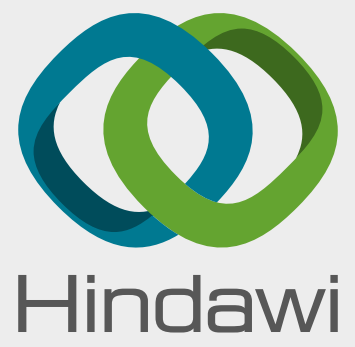

Submit your manuscripts at

www.hindawi.com
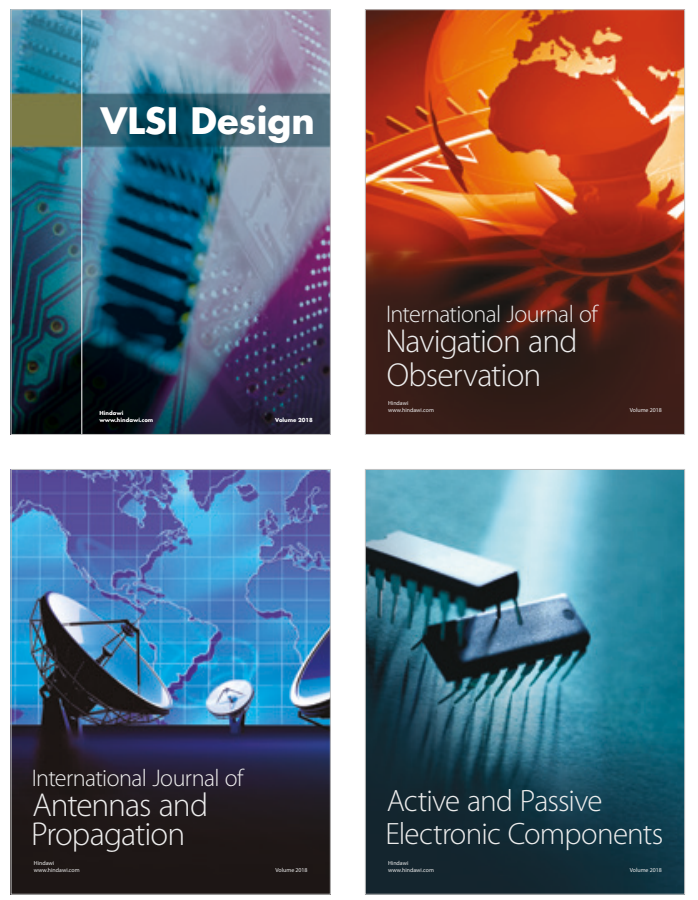
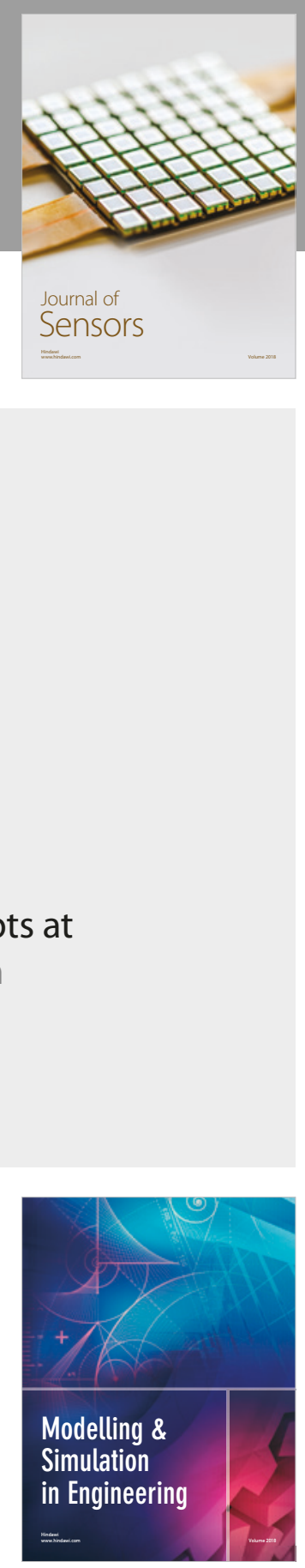

\section{Advances \\ Multimedia}
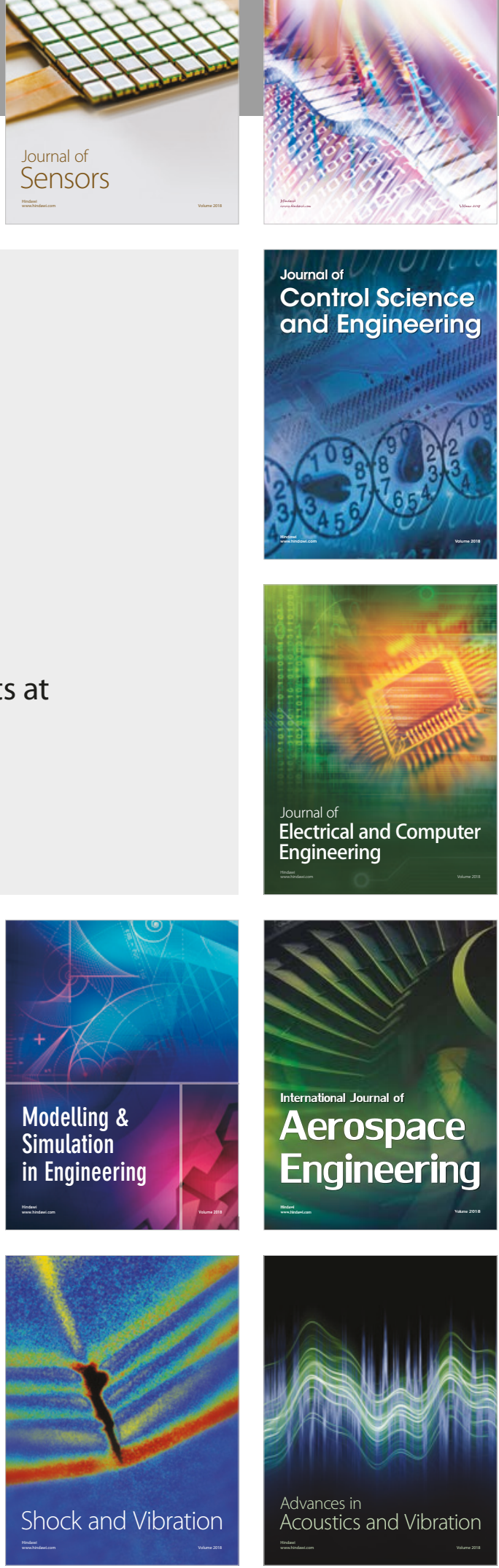